\title{
Assessment of Polycyclic Aromatic Hydrocarbons in Agricultural Soils of Teskelewu Community and Environs Impacted By Gas Flaring in Delta State, Nigeria
}

\author{
${ }^{1,2}$ ENUNEKU, AA; ${ }^{3,4}$ KUBEYINJE, B \\ ${ }^{I}$ Department of Environmental Management and Toxicology, Faculty of Life Sciences, University of Benin City, Nigeria. \\ ${ }^{2}$ Laboratory for Ecotoxicology and Environmental Forensics, University of Benin, Nigeria. \\ ${ }^{3}$ Department of Animal and Environmental Biology, University of Benin, PMB 1154, Benin City, Nigeria. \\ ${ }^{4}$ National Center for Energy and Environment, (NCEE), University of Benin, Benin City \\ *Corresponding Author Email: alex.enuneku@uniben.edu; Tel: +2348030773314
}

\begin{abstract}
Polycyclic aromatic hydrocarbons (PAHs) are emerging ubiquitous environmental pollutants with reported carcinogenic, mutagenic and teratogenic potentials on living organisms and humans. The objective of this study was to assess the concentrations of PAHs in agricultural soils of Tsekelewu Community and environs (Egbema Kingdom) in Warri North Local Government Area of Delta State, Nigeria impacted by gas flaring using gas chromatograph system Agilent 5890 Series 11 gas chromatograph equipped with Flame ionization detector (GC-FID). The results revealed that station 1 and 2 were fairly contaminated; with mean total PAH concentration of $236.40 \mu \mathrm{g} / \mathrm{kg}$ and $279.75 \mu \mathrm{g} / \mathrm{kg}$ respectively. Stations 3, 4, 6, 7 and 9 having mean total PAH concentration of $692.45 \mu \mathrm{g} / \mathrm{kg}, 726.22 \mu \mathrm{g} / \mathrm{kg}, 739.82 \mu \mathrm{g} / \mathrm{kg}$, $609.38 \mu \mathrm{g} / \mathrm{kg}$, and $772.99 \mu \mathrm{g} / \mathrm{kg}$ were moderately contaminated. Heavy contamination was recorded in station 5 and station 8 with mean PAH concentrations of $1231.08 \mu \mathrm{g} / \mathrm{kg}$ and $1224.33 \mu \mathrm{g} / \mathrm{kg}$. The dominant PAHs in soil samples were benzo(a)anthracene, phananthrene and chrysene with benzo(a)anthracene having the highest concentration across all sample stations. The PAH concentration observed in this study is thus a serious cause for concern since soil contamination from gas flaring will likely continue. Measures that will attenuate PAHs concentrations in soil should be adopted as farmers are likely exposed by largely inhalation.
\end{abstract}

\section{DOI: https://dx.doi.org/10.4314/jasem.v23i10.15}

Copyright: Copyright $(\subset) 2019$ Enuneku and Kubeyinje. This is an open access article distributed under the Creative Commons Attribution License (CCL), which permits unrestricted use, distribution, and reproduction in any medium, provided the original work is properly cited.

Dates: Received: 20 March 2019; Revised: 25 October 2019; 29 October 2019

Keywords: Hydrocarbons; Contamination; Agricultural soils; Gas flaring

Polycyclic aromatic hydrocarbons (PAHs) have become ubiquitous environmental pollutants thereby attracting the interest of the researchers all over the world. This increased interest emanates from the reported carcinogenic, mutagenic and teratogenic potentials of PAHs on living organisms and humans (Olsson et al., 2010; Zheng et al. 2012). PAHs are a group of semi-volatile organic compounds that are composed of two or more aromatic (benzene) rings fused together in a variety of configurations (Doherty et al., 2015). Generally, PAHs are characterized by high lipid solubility, facile bioaccumulation, environmental toxicity, and persistent nature, with high melting and boiling points and low vapor pressure (Akyuz and Cabuk, 2010; Abdel-Shafy et al., 2016). Formation of PAH is due to incomplete combustion of organic matter through the condensation of ethylenic radicals in the gas phase to form the larger polycyclic compounds (Lane, 1989). PAHs may originate from natural sources (PAH background values) such as forest fires, volcanic activity or as a result of anthropogenic activities like incomplete combustion of fossil fuels and industrial emissions (Al-Saad et al., 2019). PAHs found in the urban environment are predominantly anthropogenic in origin. They may be either associated with (a) petroleum products introduced to the environment through spills and industrial discharges (generally termed "petrogenic") or (b) the incomplete combustion of organic materials emitted by various engine types from automobiles to power plants (termed "pyrogenic"). Depending on their molecular structure and number of aromatic rings, PAHs can be grouped into low-molecular weight PAHs (LMWPAHs), with two or three aromatic rings, and high-molecular weight PAHs (HMWPAHs), with four or more aromatic rings (Gereslassie et al., 2018). Low-molecular weight (LMWPAHs) are environmentally abundant, occur in the atmosphere and are highly toxic compounds. However, they are relatively less persistent, have lower carcinogenicity, and are more easily degradable than high-molecular weight (HMWPAHs) (Kumar et al., 2012). Low molecular weight alkyl PAHs generally indicate petrogenic origin (alkylated PAHs), whereas high molecular weight generally indicates pyrogenic origin (parent PAHs). The ratio of similar mass PAH isomers can give an indication of their likely source (Doherty et al., 2015). PAH exhibit their mutagenic and carcinogenic activity through biotransformation to chemically reactive intermediates which bind covalently to cellular macromolecules (inter alia DNA) (WHO, 2000). PAHs are prone to enrichment in the soil matrix long after being adsorbed by the soil and sediment organic matter and more than $90 \%$ of the total mass of PAHs in the environment is stored in soils (Bergamasco et al., 2015; Bi et al., 
2016). Therefore soil is considered as the final reservoir and repository for such hydrophobic organic contaminants (Kwon and Choi 2014, Wang et al. 2015). In warmer climates, soil can contribute PAHs to the atmosphere (Dalla-Valle et al., 2005). Further, leaching of PAHs from soils can contaminate groundwater (Bortey-Sam et al., 2014). Therefore, monitoring concentrations of PAHs in soils is important for assessing their potential effects on the environment or humans. Gas flaring practices has been preferred as a means of disposing associated or waste gas by various petroleum exploration and production companies operating in the Nigeria's Niger Delta for the past five decades (Ite and Ibok, 2013). This practice has enormous impact on the soil, water and atmospheric conditions of the immediate environment. Assessment of the PAH compound ratios, phenanthrene/anthracene and fluoranthene/pyrene, suggested that predominant PAHs of pyrogenic sources on surface soils is an indication that oil leakage and/or gas flaring contributes to soil contamination (Sojinu et al., 2010). Gas flaring in Tsekelewu Community is thus a threat to the inhabitants of the community due to the release of associated contaminants such as benzene, toluene, ethyl benzene, and xylenes (BTEX), aliphatic and polycyclic aromatic hydrocarbons (PAHs) which ultimately deposit in the soil. Assessment of the level of contamination in these soils is thus a necessity.

The aim of this study is to assess of concentrations of PAHs in agricultural soils of Tsekelewu Community impacted by gas flaring in Warri North Local Government Area of Delta State.

\section{MATERIALS AND METHODS}

Study Area: The study was carried in Oil rich Tsekelewu community and environs in Delta State, Southern Nigeria. The nine communities involved were Tsekelewu - Station 1 (Long. N05.97527 ${ }^{\circ}$, Lat. E004.97356 $)$; Ikpoku-gbene - Station 2 (Long.N05.94935 ${ }^{\circ}$, Lat. E005.01663 ${ }^{\circ}$ ); Iralatei Station 3 (Long.N05.94627 ${ }^{\circ}$, Lat. E005.02834 ${ }^{\circ}$ ); Agoduba - Station $4\left(\mathrm{~N} 05.94014^{\circ}\right.$, E005.04122 ${ }^{\circ}$ ); Ozuoedodo - Station 5 (Long.N05.94054, Lat. E005.04335 ); Weke-gbene - Station 6 (Long N05.93746 ${ }^{\circ}$, Lat. E005.04745), Opuama - Station 7 (Long.N05.91754, Lat. E005.05929 ${ }^{\circ}$ ); Ayara-Gbene Station 8 (Long N05.92470 ${ }^{\circ}$, Lat. E005.02323 ${ }^{\circ}$ ) and Tangege-gbene - Station 9 (Long. N05.93379 ${ }^{\circ}$, Lat. E005.03247 ${ }^{\circ}$. The map of the study area (Figure 1) was created with QGIS software (Version 3.6.1) using satellite imagery.

Sample Collection: An initial survey was carried out on the site prior to sample collection in order to ensure that challenges during sampling would be minimized. Surface soil samples were collected from agricultural soils of Tsekelewu community and environs that are subjected to gas flaring activities. Samples were collected using a hand held trowel that was washed and dried after collection from each station. The samples were placed in polyethylene plastic bags, properly labeled and transported on an ice chest to the laboratory where it was stored in a chiller at $4^{\circ} \mathrm{C}$.

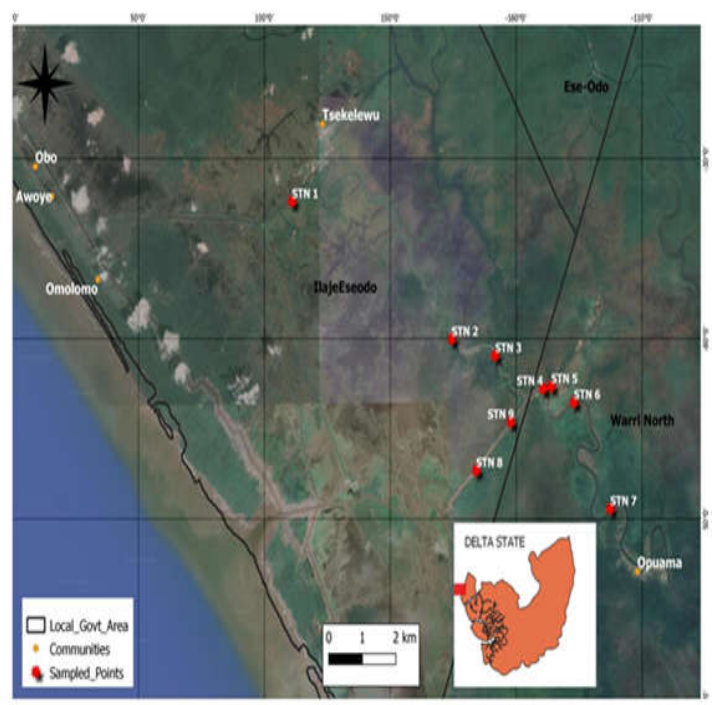

Fig 1: Map showing the stations sampled in the study area

Soil Sample Extraction: The extraction of soil sample was carried out by weighing $5 \mathrm{~g}$ each soil sample into pre-cleaned $25 \mathrm{ml}$ amber glass bottles. $10 \mathrm{ml}$ of hexane was added, respectively. The bottles were sealed with a screw cap closure lined with a PTFE-faced silicone rubber septum and shaken vigorously to suspend the contents. The bottles were then sonicated in a high performance ultrasonic bath with microprocessor control for precision time and temperature controlled operation for $30 \mathrm{~min}$. the sample bottles were intermittently inverted and shaken to continually resuspend the samples after which the sample was filtered. After filtration the solvent was evaporated/blown down under gentle steam of oxygen to $0.5 \mathrm{ml}$.

Clean up: One $\mathrm{cm}$ of moderately packed glass wool was placed at the bottom of $10 \mathrm{~mm}$ ID $\times 250$ Loup chromatographic column. Then $3.5 \mathrm{~g}$ silica gel was poured into the chromatographic column. To the top was added $0.5 \mathrm{~cm}$ of sodium sulphate. The $0.5 \mathrm{ml}$ extracted sample was poured into the column. The extraction bottle was rinsed with $7 \mathrm{ml}$ of hexane and added to the column immediately. The effluent/extract was collected with a $10 \mathrm{ml}$ bottle and evaporated/blown down under gentle steam of oxygen to $0.2 \mathrm{ml}$. The aromatic concentrates were transferred into labeled $1.5 \mathrm{ml}$ glass vials and hexane was added up to $0.5 \mathrm{ml}$. The vials were capped with Teflon rubber crimp and sealed in preparation for the GC analysis.

PAHs analysis: The determination of PAHs was performed on a high resolution gas chromatograph system Agilent 5890 Series 11 gas chromatograph equipped with Flame ionization detector (GC-FID), 
with on-column capillary injection system and Hewlett-Packard model 7673 autos ampler. For the PAHs determination, 0.5 micro-litre of each sample was injected by the auto-sampler a splitless mode. The separation was performed on fused silica capillary columns DB-5, $30 \mathrm{~m} \times 0.25 \mathrm{~mm}$ internal diameter, and $0.25 \mu \mathrm{m}$ film thickness (5\% phenyl silicone, 95\% methyl silicone). High purity nitrogen was used as carrier gas at flow rate of $1 \mathrm{~mL} / \mathrm{min}$ and $30 \mathrm{~mL} / \mathrm{min}$ respectively. The oven temperature programmed was as follows: initial temperature of $80^{\circ} \mathrm{C}$ was held for 2 min, increased to $190{ }^{\circ} \mathrm{C}$ at $5{ }^{\circ} \mathrm{C} / \mathrm{min}$, and then increased to $280{ }^{\circ} \mathrm{C}$ at $10{ }^{\circ} \mathrm{C} / \mathrm{min}$. The injector and detector temperatures were set at $250{ }^{\circ} \mathrm{C}$ and $300{ }^{\circ} \mathrm{C}$, respectively. Identification of the individual PAHs was based on comparison of retention time between samples and the standard solution. Quantification was performed by five-point calibration method (from 100 to500 $\mathrm{ng} / \mathrm{mL}, \mathrm{r}^{2}>0.992$ ) in all cases.

\section{RESULTS AND DISCUSSION}

The summary of PAH concentration in the study stations are shown in Table 1. Mean ranges of the 16 PAHs in this study werenaphthalene $(0.00-246.07$ $\mu \mathrm{g} / \mathrm{kg})$, Acenaphthene $(0.67-153.55 \mu \mathrm{g} / \mathrm{kg})$ Acenaphthylene $(0.00-80.91 \mathrm{mg} / \mathrm{kg})$, Flourene $(0.00$ $-101.67 \mathrm{mg} / \mathrm{kg})$, Phenanthrene $(0.00-714.28 \mu \mathrm{g} / \mathrm{kg})$, anthracene $(0.00-31.41 \mu \mathrm{g} / \mathrm{kg})$, flouranthene $(0.00-$ $331.60 \mu \mathrm{g} / \mathrm{kg})$, pyrene $(0.00-167.54 \mu \mathrm{g} / \mathrm{kg})$, Chrysene $(1.05-812.36 \mu \mathrm{g} / \mathrm{kg})$, Benzo (a) anthracene $(0.35-1146.65 \mu \mathrm{g} / \mathrm{kg})$, Benzo (b) flouranthene $(0.00$ $-15.77 \mu \mathrm{g} / \mathrm{kg})$, Benzo (k) flouranthene $(0.84-18.08$ $\mu \mathrm{g} / \mathrm{kg})$, Benzo (a) pyrene $(0.00-35.68 \mu \mathrm{g} / \mathrm{kg})$, indeno $(1,2,3-\mathrm{d})$ pyrene $(0.00-64.63 \mu \mathrm{g} / \mathrm{kg})$ Dibenzo $(\mathrm{a}, \mathrm{h})$ anthracene $(0.00$ to $62.67 \mu \mathrm{g} / \mathrm{kg})$, and Benzo $(\mathrm{g}, \mathrm{h})$ perylene $(0.00-2165.74 \mu \mathrm{g} / \mathrm{kg})$. Mean concentration of the 16 PAHs in all the sampled stations varied between $14.78 \pm 29.37 \mu \mathrm{g} / \mathrm{kg}$ to $76.94 \pm 191.40 \mu \mathrm{g} / \mathrm{kg}$. The highest $(1231.08 \mu \mathrm{g} / \mathrm{kg})$ sum concentration of PAH were observed in station 5 while the lowest $(236.40 \mu \mathrm{g} / \mathrm{kg})$ was observed in station 1. Pearson correlation between the PAH concentration is shown in Table 2. Significant positive correlations were observed between Acy/Ace (0.833), Fln/Ace (0.864), Fln/Acy (0.985), Phe/Ace (0.892), Phe/Acy (0.984), Phe/Fln (0.993), Ant/Ace (0.862), Ant/Acy (0.965), Ant/Flu (0.992), Ant/Phe (0.977), Flu/Ace (0.910), Flu/Acy (0.822), Flu/Fln (0.890), Pyr/Ace (0.827), $\mathrm{Pyr} / \mathrm{Fln}$ (0.634), Pyr/Phe (0.624), Pyr/Ant (0.692), Pyr/Flu (0.908), BbF/Ace (0.740), Bbf/Flu (0.621), Bbf/Pyr (0.763), BaP/Fln (0.624), BaP/Phe (0.662), $\mathrm{BaP} / \mathrm{Ant}$ (0.632), BaP/Flu (0.787) and $\mathrm{BaP} / \mathrm{Pyr}$ (0.850).

Table 1: Summary statistics of PAHs in agricultural soils of Tsekelewu Community and environs

\begin{tabular}{|c|c|c|c|c|c|c|}
\hline & \multirow[t]{2}{*}{ PAH } & \multicolumn{3}{|c|}{ Stations } & \multicolumn{2}{|l|}{ Class } \\
\hline & & Station 1 & Station 2 & Station 3 & Station 4 & \\
\hline Naphthalene & $\mathrm{NaP}$ & $\begin{array}{l}9.66 \pm 16.73 \\
(0.00-28.97)\end{array}$ & $\begin{array}{l}82.02 \pm 142.07 \\
(0.00-246.07)\end{array}$ & $\begin{array}{l}0.98 \pm 1.70 \\
(0.00-2.94)\end{array}$ & $\begin{array}{l}72.78 \pm 126.06 \\
(0.00-218.34)\end{array}$ & $2 \mathrm{~B}$ \\
\hline Acenaphthene & Ace & $\begin{array}{l}9.7413 .12 \\
(0.67-24.78)\end{array}$ & $\begin{array}{l}8.46 \pm 6.29 \\
(1.37-13.37)\end{array}$ & $\begin{array}{l}12.79 \pm 14.60 \\
(2.15-29.44)\end{array}$ & $\begin{array}{l}8.55 \pm 6.82 \\
(2.88-16.11)\end{array}$ & $\mathrm{NC}$ \\
\hline Acenaphthylene & Acy & $\begin{array}{l}1.18 \pm 2.04 \\
(0.00-3.54)\end{array}$ & $\begin{array}{l}1.45 \pm 2.52 \\
(0.00-4.36)\end{array}$ & $\begin{array}{l}2.75 \pm 4.76 \\
(0.00-8.25)\end{array}$ & $\begin{array}{l}1.92 \pm 3.33 \\
(0.00-5.76)\end{array}$ & $\mathrm{NC}$ \\
\hline Flourene & Fln & $\begin{array}{l}2.69 \pm 4.66 \\
(0.00-8.08)\end{array}$ & $\begin{array}{l}2.83 \pm 4.89 \\
(0.00-8.48)\end{array}$ & $\begin{array}{l}7.87 \pm 13.64 \\
(0.00-23.62)\end{array}$ & $\begin{array}{l}4.98 \pm 8.63 \\
(0.00-14.95)\end{array}$ & $\mathrm{NC}$ \\
\hline Phenanthrene & Phe & $\begin{array}{l}27.59 \pm 47.79 \\
(0.00-82.78)\end{array}$ & $\begin{array}{l}9.27 \pm 16.05 \\
(0.00-27.80)\end{array}$ & $\begin{array}{l}33.28 \pm 57.65 \\
(0.00-99.85)\end{array}$ & $\begin{array}{l}28.87 \pm 50.00 \\
(0.00-86.60)\end{array}$ & $\mathrm{NC}$ \\
\hline Anthracene & Ant & $\begin{array}{l}0.98 \pm 1.70 \\
(0.00-2.95)\end{array}$ & $\begin{array}{l}0.75 \pm 1.30 \\
(0.00-2.25)\end{array}$ & $\begin{array}{l}3.74 \pm 6.48 \\
(0.00-11.23)\end{array}$ & $\begin{array}{l}1.33 \pm 2.31 \\
(0.00-3.40)\end{array}$ & $\mathrm{NC}$ \\
\hline Flouranthene & Flu & $\begin{array}{l}19.47 \pm 33.72 \\
(0.00-58.41)\end{array}$ & $\begin{array}{l}11.95 \pm 20.70 \\
(0.00-35.85)\end{array}$ & $\begin{array}{l}74.89 \pm 129.72 \\
(0.00-224.68)\end{array}$ & $\begin{array}{l}16.67 \pm 28.87 \\
(0.00-50.00)\end{array}$ & $\mathrm{NC}$ \\
\hline Pyrene & Pyr & $\begin{array}{l}9.05 \pm 15.67 \\
(0.00-27.14)\end{array}$ & $\begin{array}{l}5.22 \pm 9.03 \\
(0.00-15.65)\end{array}$ & $\begin{array}{l}39.70 \pm 68.77 \\
(0.00-119.11)\end{array}$ & $\begin{array}{l}6.19 \pm 10.72 \\
(0.00-18.60)\end{array}$ & $\mathrm{NC}$ \\
\hline Chrysene & Chr & $\begin{array}{l}24.16 \pm 33.25 \\
(4.80-62.56)\end{array}$ & $\begin{array}{l}13.78 \pm 18.02 \\
(1.05-34.40)\end{array}$ & $\begin{array}{l}38.67 \pm 62.90 \\
(1.24-111.29)\end{array}$ & $\begin{array}{l}279.57 \pm 461.53 \\
(2.77-812.36)\end{array}$ & $2 \mathrm{~B}$ \\
\hline Benzo (a) anthracene & $\mathrm{BaA}$ & $\begin{array}{l}119.99 \pm 200.38 \\
(3.99-351.36)\end{array}$ & $\begin{array}{l}112.95 \pm 186.51 \\
(0.35-328.24)\end{array}$ & $\begin{array}{l}470.99 \pm 509.90 \\
(1.35-719.50)\end{array}$ & $\begin{array}{l}297.09 \pm 509.90 \\
(1.32-885.87)\end{array}$ & $\mathrm{B}$ \\
\hline Benzo (b)fluoranthene & $\mathrm{BbF}$ & $\begin{array}{l}0.51 \pm 0.88 \\
(0.00-1.53)\end{array}$ & $\begin{array}{l}0.13 \pm 0.22 \\
(0.00-0.38)\end{array}$ & $\begin{array}{l}0.34 \pm 0.59 \\
(0.00-1.02)\end{array}$ & $\begin{array}{l}0.05 \pm 0.08 \\
(0.00-0.14)\end{array}$ & $2 \mathrm{~B}$ \\
\hline Benzo (k) fluoranthene & $\mathrm{BkF}$ & $\begin{array}{l}2.86 \pm 2.52 \\
(0.95-5.71)\end{array}$ & $\begin{array}{l}7.28 \pm 5.11 \\
(1.60-11.50)\end{array}$ & $\begin{array}{l}4.17 \pm 2.40 \\
(1.40-5.66)\end{array}$ & $\begin{array}{l}4.63 \pm 3.88 \\
(1.15-8.81)\end{array}$ & $2 \mathrm{~B}$ \\
\hline Benzo (a) pyrene & $\mathrm{BaP}$ & $\begin{array}{l}0.33 \pm 0.57 \\
(0.00-0.98)\end{array}$ & $\begin{array}{l}0.33 \pm 0.57 \\
(0.00-0.99)\end{array}$ & $\begin{array}{l}0.93 \pm 1.60 \\
(0.00-2.78)\end{array}$ & $\begin{array}{l}0.38 \pm 0.65 \\
(0.00-1.13)\end{array}$ & $\mathrm{B}$ \\
\hline Indeno(1,2,3-cd)pyrene & icdP & $\begin{array}{l}4.06 \pm 5.12 \\
(0.00-9.81)\end{array}$ & $\begin{array}{l}11.45 \pm 14.41 \\
(0.00-27.63)\end{array}$ & $\begin{array}{l}0.68 \pm 1.17 \\
(0.00-2.02)\end{array}$ & $\begin{array}{l}2.15 \pm 0.37 \\
(0.00-0.64)\end{array}$ & $2 \mathrm{~B}$ \\
\hline Dibenzo(a,h)anthracene & $\mathrm{DaA}$ & $\begin{array}{l}1.93 \pm 3.35 \\
(0.00-5.80)\end{array}$ & $\begin{array}{l}4.31 \pm 5.07 \\
(0.00-9.90)\end{array}$ & $\begin{array}{l}0.39 \pm 0.68 \\
(0.00-1.18)\end{array}$ & $\begin{array}{l}0.38 \pm 0.66 \\
(0.00-1.14)\end{array}$ & B \\
\hline Benzo $(\mathrm{g}, \mathrm{h}, \mathrm{i})$ perylene & $\mathrm{BgP}$ & $\begin{array}{l}2.20 \pm 3.82 \\
(0.00-6.61)\end{array}$ & $\begin{array}{l}7.57 \pm 12.34 \\
(0.00-21.82)\end{array}$ & $\begin{array}{l}0.28 \pm 0.49 \\
(0.00-0.85)\end{array}$ & $\begin{array}{l}0.68 \pm 1.18 \\
(0.00-2.04)\end{array}$ & $\mathrm{NC}$ \\
\hline & $\begin{array}{r}\Sigma 16 \\
\bar{X}\end{array}$ & $\begin{array}{l}236.40 \\
14.78 \pm 29.37\end{array}$ & $\begin{array}{l}279.75 \\
17.48 \pm 32.02\end{array}$ & $\begin{array}{l}692.45 \\
43.28 \pm 116.02\end{array}$ & $\begin{array}{l}726.22 \\
45.39 \pm 96.62\end{array}$ & \\
\hline
\end{tabular}

PAHS concentrations are in $\mu \mathrm{g} / \mathrm{Kg}$. Values are expressed as mean \pm standard deviation. 
Table 1: Continue

\begin{tabular}{|c|c|c|c|c|c|c|c|}
\hline & \multirow[t]{2}{*}{ PAH } & \multicolumn{4}{|c|}{ Stations } & \multicolumn{2}{|l|}{ Class } \\
\hline & & Station 5 & Station 6 & Station 7 & Station 8 & Station 9 & \\
\hline Naphthalene & $\mathrm{NaP}$ & $\begin{array}{l}75.93 \pm 131.51 \\
(0.00-227.78)\end{array}$ & $\begin{array}{l}74.63 \pm 129.26 \\
(0.00-223.88)\end{array}$ & $\begin{array}{l}45.01 \pm 77.95 \\
(0.00-135.02)\end{array}$ & $\begin{array}{l}18.95 \pm 32.82 \\
(0.00-56.84)\end{array}$ & $\begin{array}{l}13.53 \pm 23.43 \\
(0.00-40.59)\end{array}$ & $2 \mathrm{~B}$ \\
\hline Acenaphthene & Ace & $\begin{array}{l}14.20 \pm 13.62 \\
(6.17-29.82)\end{array}$ & $\begin{array}{l}12.69 \pm 14.86 \\
(2.63-29.75)\end{array}$ & $\begin{array}{l}9.20 \pm 4.97 \\
(4.18-14.12)\end{array}$ & $\begin{array}{l}55.03 \pm 85.30 \\
(4.30-153.52)\end{array}$ & $\begin{array}{l}53.18 \pm 86.93 \\
(1.69-153.55)\end{array}$ & $\mathrm{NC}$ \\
\hline Acenaphthylene & Acy & $\begin{array}{l}0.22 \pm 0.37 \\
(0.00-0.65)\end{array}$ & $\begin{array}{l}1.99 \pm 3.43 \\
(0.00-5.96)\end{array}$ & $\begin{array}{l}0.48 \pm 0.83 \\
(0.00-1.45)\end{array}$ & $\begin{array}{l}26.97 \pm 46.71 \\
(0.00-80.91)\end{array}$ & $\begin{array}{l}6.51 \pm 11.27 \\
(0.00-19.53)\end{array}$ & $\mathrm{NC}$ \\
\hline Flourene & Fln & $\begin{array}{l}2.81 \pm 4.87 \\
(0.00-8.43)\end{array}$ & $\begin{array}{l}5.17 \pm 8.96 \\
(0.00-15.51)\end{array}$ & $\begin{array}{l}1.61 \pm 2.78 \\
(0.00-4.82)\end{array}$ & $\begin{array}{l}33.89 \pm 58.70 \\
(0.00-101.67)\end{array}$ & $\begin{array}{l}10.59 \pm 18.34 \\
(0.00-31.76)\end{array}$ & $\mathrm{NC}$ \\
\hline Phenanthrene & Phe & $\begin{array}{l}24.11 \pm 41.76 \\
(0.00-72.33)\end{array}$ & $\begin{array}{l}39.84 \pm 69.01 \\
(0.00-119.53)\end{array}$ & $\begin{array}{l}13.07 \pm 22.63 \\
(0.00-39.20)\end{array}$ & $\begin{array}{l}238.09 \pm 412.39 \\
(0.00-714.28)\end{array}$ & $\begin{array}{l}85.52 \pm 148.13 \\
(0.00-256.57)\end{array}$ & $\mathrm{NC}$ \\
\hline Anthracene & Ant & $\begin{array}{l}0.86 \pm 1.49 \\
(0.00-2.59)\end{array}$ & $\begin{array}{l}1.53 \pm 2.65 \\
(0.00-4.60)\end{array}$ & $\begin{array}{l}0.09 \pm 0.16 \\
(0.00-0.27)\end{array}$ & $\begin{array}{l}10.47 \pm 18.14 \\
(0.00-31.41)\end{array}$ & $\begin{array}{l}3.57 \pm 6.17 \\
(0.00-10.70)\end{array}$ & $\mathrm{NC}$ \\
\hline Flouranthene & Flu & $\begin{array}{l}7.34 \pm 12.72 \\
(0.00-22.03)\end{array}$ & $\begin{array}{l}28.84 \pm 49.95 \\
(0.00-86.52)\end{array}$ & $\begin{array}{l}0.97 \pm 1.67 \\
(0.00-2.90)\end{array}$ & $\begin{array}{l}110.53 \pm 191.45 \\
(0.00-331.60)\end{array}$ & $\begin{array}{l}89.32 \pm 154.71 \\
(0.00-267.97)\end{array}$ & $\mathrm{NC}$ \\
\hline Pyrene & Pyr & $\begin{array}{l}4.50 \pm 7.80 \\
(0.00-13.51)\end{array}$ & $\begin{array}{l}10.79 \pm 18.68 \\
(0.00-32.36)\end{array}$ & $\begin{array}{l}6.13 \pm 10.61 \\
(0.00-18.39)\end{array}$ & $\begin{array}{l}26.65 \pm 46.17 \\
(0.0079 .96)\end{array}$ & $\begin{array}{l}55.84 \pm 96.73 \\
(0.00-167.54)\end{array}$ & $\mathrm{NC}$ \\
\hline Chrysene & Chr & $\begin{array}{l}12.77 \pm 13.35 \\
(3.95-28.13)\end{array}$ & $\begin{array}{l}295.66 \pm 392.44 \\
(57.47-748.61)\end{array}$ & $\begin{array}{l}33.78 \pm 40.48 \\
(6.63-80.31)\end{array}$ & $\begin{array}{l}39.42 \pm 60.26 \\
(1.63-108.91)\end{array}$ & $\begin{array}{l}35.55 \pm 56.31 \\
(1.57-100.55)\end{array}$ & 2B \\
\hline $\begin{array}{l}\text { Benzo } \\
\text { anthracene }\end{array}$ & $\mathrm{BaA}$ & $\begin{array}{l}338.79 \pm 338.05 \\
(0.82-676.93)\end{array}$ & $\begin{array}{l}257.82 \pm 443.94 \\
(0.61-770.43)\end{array}$ & $\begin{array}{l}471.17 \pm 412.12 \\
(0.66-768.15)\end{array}$ & $\begin{array}{l}591.47 \pm 572.76 \\
(2.62-1146.65)\end{array}$ & $\begin{array}{l}389.05 \pm 426.74 \\
(11.21-851.90)\end{array}$ & B \\
\hline $\begin{array}{l}\text { Benzo } \\
\text { (b)fluoranthene }\end{array}$ & $\mathrm{BbF}$ & $\begin{array}{l}1.44 \pm 2.49 \\
(0.00-4.31)\end{array}$ & $\begin{array}{l}2.70 \pm 4.67 \\
(0.00-8.09)\end{array}$ & $\begin{array}{l}0.12 \pm 0.21 \\
(0.00-0.37)\end{array}$ & $\begin{array}{l}0.87 \pm 1.51 \\
(0.00-2.61)\end{array}$ & $\begin{array}{l}5.25 \pm 9.10 \\
(0.00-15.77)\end{array}$ & 2B \\
\hline $\begin{array}{l}\text { Benzo } \\
\text { fluoranthene }\end{array}$ & $\mathrm{BkF}$ & $\begin{array}{l}4.09 \pm 2.01 \\
(2.64-6.38)\end{array}$ & $\begin{array}{l}5.30 \pm 0.90 \\
(4.27-5.91)\end{array}$ & $\begin{array}{l}4.58 \pm 2.25 \\
(2.02-6.20)\end{array}$ & $\begin{array}{l}4.53 \pm 3.19 \\
(0.84-6.38)\end{array}$ & $\begin{array}{l}8.25 \pm 8.51 \\
(3.16-18.08)\end{array}$ & $2 \mathrm{~B}$ \\
\hline Benzo (a) pyrene & $\mathrm{BaP}$ & $\begin{array}{l}1.33 \pm 2.31 \\
(0.00-4.00)\end{array}$ & $\begin{array}{l}0.13 \pm 0.22 \\
(0.00-0.38)\end{array}$ & $\begin{array}{l}0.32 \pm 0.56 \\
(0.00-0.97)\end{array}$ & $\begin{array}{l}5.54 \pm 9.61 \\
(0.00-16.64)\end{array}$ & $\begin{array}{l}11.89 \pm 20.60 \\
(0.00-35.68)\end{array}$ & B \\
\hline $\begin{array}{l}\text { Indeno(1,2,3- } \\
\text { cd)pyrene }\end{array}$ & icdP & $\begin{array}{l}19.79 \pm 33.07 \\
(0.00-57.96)\end{array}$ & $\begin{array}{l}0.92 \pm 1.59 \\
(0.00-2.76)\end{array}$ & $\begin{array}{l}13.95 \pm 20.67 \\
(0.00-37.70)\end{array}$ & $\begin{array}{l}23.32 \pm 35.88 \\
(0.00-64.63)\end{array}$ & $\begin{array}{l}1.71 \pm 2.96 \\
(0.00-5.13)\end{array}$ & 2B \\
\hline $\begin{array}{l}\text { Dibenzo(a,h)anth } \\
\text { racene }\end{array}$ & $\mathrm{DaA}$ & $\begin{array}{l}0.25 \pm 0.43 \\
(0.00-0.74)\end{array}$ & $\begin{array}{l}1.48 \pm 2.56 \\
(0.00-4.45)\end{array}$ & $\begin{array}{l}0.41 \pm 0.72 \\
(0.00-1.24)\end{array}$ & $\begin{array}{l}23.02 \pm 34.49 \\
(0.00-62.67)\end{array}$ & $\begin{array}{l}1.44 \pm 2.50 \\
(0.00-4.33)\end{array}$ & B \\
\hline $\begin{array}{l}\text { Benzo } \quad(g, h, i) \\
\text { perylene }\end{array}$ & $\begin{array}{r}\mathrm{BgP} \\
\mathbf{\Sigma 1 6} \\
\overline{\boldsymbol{X}} \\
\end{array}$ & $\begin{array}{l}\mathbf{7 2 2 . 6 5} \pm 1249.76 \\
(0.00-2165.74) \\
\mathbf{1 2 3 1 . 0 8} \\
\mathbf{7 6 . 9 4} \pm \mathbf{1 9 1 . 4 0}\end{array}$ & $\begin{array}{l}0.33 \pm 0.57 \\
(0.00-0.98) \\
\mathbf{7 3 9 . 8 2} \\
\mathbf{4 6 . 2 4} \pm \mathbf{9 2 . 3 1 3}\end{array}$ & $\begin{array}{l}8.49 \pm 11.29 \\
(0.00-21.28) \\
\mathbf{6 0 9 . 3 8} \\
\mathbf{3 8 . 0 9} \pm \mathbf{1 1 6 . 2 0}\end{array}$ & $\begin{array}{l}15.58 \pm 18.78 \\
(0.00-36.44) \\
\mathbf{1 2 2 4 . 3 3} \\
\mathbf{7 6 . 5 2} \pm \mathbf{1 4 9 . 2 3}\end{array}$ & $\begin{array}{l}1.79 \pm 2.28 \\
(0.00-4.36) \\
\mathbf{7 7 2 . 9 9} \\
\mathbf{4 8 . 3 1} \pm \mathbf{9 5 . 6 1}\end{array}$ & $\mathrm{NC}$ \\
\hline
\end{tabular}

Over a thousand PAH compounds have been defined but the United States Environmental Protection Agency (USEPA) classified 16 PAHs as priority pollutants and eight as carcinogens. (Liu et al., 2016). The Eight PAHs (Car-PAHs) typically considered as possible carcinogens are: benzo(a)anthracene, chrysene, benzo(b)fluoranthene, benzo(k)fluoranthene, benzo(a)pyrene (B(a)P), dibenzo(a,h)anthracene, indeno(1,2,3-cd)pyrene and benzo $(\mathrm{g}, \mathrm{h}, \mathrm{i})$ perylene. In particular, benzo(a)pyrene has been identified as being highly carcinogenic (Srogi, 2007). According to the European classification system of soil contamination (Maliszewska-Kordybach, 1996) $\Sigma 16$ PAHs $<0.20$ $\mathrm{mg} / \mathrm{kg}$ show no contamination, $0.20-0.60 \mathrm{mg} / \mathrm{kg}$ corresponds to weak contamination, $0.60-1.0 \mathrm{mg} / \mathrm{kg}$ represents moderate contamination, and $>1.0 \mathrm{mg} / \mathrm{kg}$ indicates heavy contamination.

Table 2: Inter-Item Correlation Matrix

\begin{tabular}{|c|c|c|c|c|c|c|c|c|c|c|c|c|c|c|c|c|}
\hline & $\mathbf{N a P}$ & Ace & Acy & Fln & Phe & Ant & Flu & Pyr & Chr & $\mathbf{B a A}$ & BbF & BkF & BaP & icdP & DaA & $\mathrm{BgP}$ \\
\hline$\overline{\mathrm{NaP}}$ & 1.000 & & & & & & & & & & & & & & & \\
\hline Ace & .161 & 1.000 & & & & & & & & & & & & & & \\
\hline Acy & .111 & $.833 * *$ & 1.000 & & & & & & & & & & & & & \\
\hline Fln & .208 & $.864 * *$ & $.985^{* *}$ & 1.000 & & & & & & & & & & & & \\
\hline Phe & .199 & $.892 * *$ & $.984 * *$ & $.993 * *$ & 1.000 & & & & & & & & & & & \\
\hline Ant & .166 & $.862 * *$ & $.965^{* *}$ & $.992 * *$ & $.977 * *$ & 1.000 & & & & & & & & & & \\
\hline Flu & .152 & $.910 * *$ & $.822 \% *$ & $.890 * *$ & $.875^{* *}$ & $.927 * *$ & 1.000 & & & & & & & & & \\
\hline Pyr & .132 & $.827 * *$ & .533 & $.634 *$ & $.624 *$ & $.692 *$ & $.908 * *$ & 1.000 & & & & & & & & \\
\hline Chr & -.122 & -.006 & .016 & .005 & .005 & .012 & .015 & .008 & 1.000 & & & & & & & \\
\hline $\mathbf{B a A}$ & -.491 & -.356 & -.286 & -.368 & -.360 & -.372 & -.421 & -.419 & -.321 & 1.000 & & & & & & \\
\hline BbF & .312 & $.740 *$ & .303 & .386 & .437 & .396 & $.621 *$ & .763 & -.014 & -.346 & 1.000 & & & & & \\
\hline BkF & -.334 & .263 & -.120 & -.139 & -.088 & -.130 & $.065^{*}$ & .268 & -.023 & .149 & .499 & 1.000 & & & & \\
\hline BaP & .079 & $.925^{* *}$ & .585 & $.624 *$ & $.662 *$ & $.632 \%$ & $.787 *$ & .850 & .013 & -.293 & .842 & .500 & 1.000 & & & \\
\hline icdP & -.071 & .418 & .598 & .547 & .548 & .524 & .366 & .134 & -.112 & -.014 & -.001 & -.099 & .221 & 1.000 & & \\
\hline $\mathbf{D a A}$ & .012 & .047 & .045 & .036 & .037 & .032 & .024 & .004 & -.092 & .324 & .010 & .046 & .021 & .018 & 1.000 & \\
\hline BgP & -.107 & -.071 & -.059 & -.077 & -.076 & -.078 & -.090 & -.092 & -.084 & .187 & -.077 & -.097 & -.064 & .571 & -.046 & 1.00 \\
\hline
\end{tabular}

Npl-Naphthalene, Ace-Acenaphthene, Acy-Acenaphthylene, Fl-Flourene, Phe-Phenanthrene, Ant-Acenaphthene, Flu-Flouranthene, Pyr-Pyrene, Chr-Chrysene, BaA - Benzo (a) anthracene, BaF-Benzo (a) Flouranthene, BkF-Benzo (k) Flouranthene, BaP-Benzo (a) pyrene, iEP-indeno $(1,2,3-d)$ pyrene, DaA-Dibenzo $(a, h)$ anthracene, BgP-Benzo $(g, h)$ perylene. 
Following this classification, in this study, stations 1 and 2 with total PAH concentration of $236.40 \mu \mathrm{g} / \mathrm{kg}$ and $279.75 \mu \mathrm{g} / \mathrm{kg}$ were weakly contaminated. Stations 3, 4, 6, 7 and 9 were moderately contaminated having total PAH concentration of $692.45 \mu \mathrm{g} / \mathrm{kg}, 726.22$ $\mu \mathrm{g} / \mathrm{kg}, 739.82 \mu \mathrm{g} / \mathrm{kg}, 609.38 \mu \mathrm{g} / \mathrm{kg}$, and $772.99 \mu \mathrm{g} / \mathrm{kg}$. Station 5 with total PAH $(1231.08 \mu \mathrm{g} / \mathrm{kg})$ and station 8 $(1224.33 \mu \mathrm{g} / \mathrm{kg})$ were heavily contaminated with PAH. Total mean PAH concentration in stations 5 and 8 also exceeded the Department of Petroleum resources (1999) permissible limits of $1000 \mu \mathrm{gkg}^{-1}$. The dominant PAHs in soil samples were benzo(a)anthracene, phananthrene and chrysene with the carcinogenic, persistence, bioaccumulative potentials with the toxic 4-ring benzo(a)anthracene having the highest concentration across all sample stations. The ranges for mean concentrations of benzo(a)anthracene, phananthrene and chrysene were $(112.95-591.47 \mu \mathrm{g} / \mathrm{kg}), \quad(9.27-288.09 \mu \mathrm{g} / \mathrm{kg}) \quad$ and $(12.77-295.66 \mu \mathrm{g} / \mathrm{kg})$ respectively with station 8 having the highest benzo(a)anthracene contamination. The mean PAH concentration in this study are similar with values reported by Nwaichi et al., (2016) who reported total PAH range concentration of 531.661 $4613.020 \mu \mathrm{g} / \mathrm{kg}$ in their study of some oil polluted sites in Delta state. These findings indicate that the soil in the Tsekelewu community stored copious amount of $\mathrm{PAH}$ and thus potential danger is inevitable. It has been reported that PAHs in soils might be further deposited on or accumulated into vegetables or other biota via food chains (Li et al., 2008). Previous studies have shown that 3-ring PAHs are readily absorbed by maize roots but 2- and 4-6 ring PAHs are less likely to be absorbed into the plants (Banger et al., 2010. It is worthy of note that the ground water sources of this community is also at risk due to infiltration of PAH. The low solubility of PAH in water makes them easily sorbed in the organic matter of the soil instead of being solubilized in the infiltrating water thereby transporting them downwards to the ground water (Nwaichi et al., 2016).

Given that there is no threshold concentration below which carcinogenic effects of PAH does not occur, the PAH concentration observed in this study is thus a serious cause for concern. Regulatory measures need to be put in place to stop the trend of $\mathrm{PAH}$ contamination in the area inorder to avoid contamination of water sources and health risks to inhabitants of the community.

Conclusion: In this study dominant PAHs determined in soil samples collected from Agricultural soils of Tsekelewu Community and environs were benzo(a)anthracene, phananthrene and chrysene with benzo(a)anthracene having the highest concentration across all sampled stations. The PAH concentration observed in this study is thus a cause for concern since soil contamination from gas flaring will likely continue. Measures that will attenuate PAHs concentrations in soil should be adopted as farmers are likely exposed by largely inhalation.

\section{REFERENCES}

Abdel-Shafy, HI; Mansour, MSM. (2016). A review on polycyclic aromatic hydrocarbons: Source, environmental impact, effect on human health and remediation. Egypt J. Pet, 25: 107-123

Akyüz, M; Çabuk, H. (2010) Gas-particle partitioning and seasonal variation of polycyclic aromatic hydrocarbons in the atmosphere of Zonguldak, Turkey. Sci. Total Environ, 408: 5550-5558

Al-Saad, H; Farid, W; Abdul-Ameer, W. (2019). Distribution and sources of polycyclic aromatic hydrocarbons in soils along the Shatt Al-Arab River delta in southern Iraq. Soil and Water Research, 14(2): 84-93

Banger K; Toor GS; Chirenje, T; Ma, L. (2010). Polycyclic aromatic hydrocarbons in urban soils of different land uses in Miami, Florida. Soil Sediment Contam., 19:231-243

Bergamasco, A; Culotta L; De Stefano C; Orecchio S; Sammartano S; Barreca, S. (2014). Composition, distribution, and sources of polycyclic aromatic hydrocarbons in sediments of the gulf of Milazzo (Mediterranean Sea, Italy). Polycycl. Aromat. Compd. 34:397-424

Bi, X; Luo, W; Gao, J; Xum L; Guo, J; Zhang, Q; Romesh, KY; Giesy, JP; Kang, S; Boer, J. (2016) Polycyclic aromatic hydrocarbons in soils from the Central-Himalaya region: Distribution, sources, and risks to humans and wildlife. Sci. Total Environ., 556: $12-22$

Bortey-Sam, N; Ikenaka, Y; Nakayama, SM; Akoto, O; Yohannes, YB; Baidoo, E; Mizukawa, H; Ishizuka, M. (2014). Occurrence, distribution, sources and toxic potential of polycyclic aromatic hydrocarbons (PAHs) in surface soils from the Kumasi Metropolis, Ghana. Sci. Total Environ. 496: 471-478

Dalla-Valle, M; Jurado, E; Dachs, J; Sweetman, AJ; Jones, KC. (2005). The maximum reservoir capacity of soils for persistent organic pollutants: implications for global cycling. Environ. Pollut.134: 153-164

Doherty, R; Mcllwaine, R; McAnallen, L; Cox, S. (2015). Assessment of polycyclic aromatic hydrocarbons in an urban soil dataset. Environmental Foreniscs: Proceedings of the 2014 INEF Conference

DPR (1999). Department of Petroleum Resources. Environmental guidelines and standards for the petroleum industry in Nigeria, Ministry of Petroleum resources, Lagos 
Gereslassie, T; Workineh, A; Liu, X; Yan, X; Wang, J. (2018). Occurrence and Ecological and Human Health Risk Assessment of Polycyclic Aromatic Hydrocarbons in Soils from Wuhan, Central China, Int. J. Environ. Res. Public Health, 15: 1- 19

Ite, AE; Ibok, UJ. (2013). Gas Flaring and Venting Associated with Petroleum Exploration and Production in the Nigeria's Niger Delta, Am. J. Environ. Prot., 1(4): 70-77

Kumar, R; Routh, J; Ramanathan, AL; Klump, JV. (2012). Organic Geochemistry Polycyclic aromatic hydrocarbon fingerprints in the Pichavaram mangrove-Estuarine sediments, southeastern India. Org. Geochem, 53: 88-94

Kwon H; Choi S. (2014): Polycyclic aromatic hydrocarbons (PAHs) in soils from a multi-industrial city, South Korea. Sci. Total Environ, 470: 1494 1501

Lane, D. A. (1989). The fate of polycyclic aromatic compounds in the atmosphere and during sampling.In:"Chemical analysis of polycyclic aromatic compounds.” T.Vo-Dinh (Ed.), J.Wiley\& Sons.USA pp.31-58

Li, Y.T; Li, FB; Chen, JJ; Yang, GY; Wan, HF; Zhang, TB; Zeng, XD; Liu, JM. (2008). The concentrations, distribution and sources of PAHs in agricultural soils and vegetables from Shunde, Guangdong, China. Environ. Monit. Assess, 139: 61-76

Liu, MX; Yang, YY; Yun, XY; Zhang, MM; Wang, J. (2016). Occurrence, sources, and cancer risk of polycyclic aromatic hydrocarbons and polychlorinated biphenyls in agricultural soils from the Three Gorges Dam region, China. J. Soil Water Conserv. 71: 327-334

Maliszewska-Kordybach, B. (1996). Polycyclic aromatic hydrocarbons in agricultural soils in Poland: Preliminary proposals for criteria to evaluate the level of soil contamination, Appl. Geochem, 11: 121-127
Nwaichi, EO; Chuku, LC; Ighoavwogan, E. (2016). Polycyclic Aromatic Hydrocarbons and Selected Heavy Metals in Some Oil Polluted Sites in Delta State Nigeria. J. Environ. Prot., 7: 1389-1410

Olsson, AC; Fevotte, J; Fletcher, T; Cassidy, A; Mannetje, A; Zaridze, D; Szeszenia-Dabrowska, N; Rudnai, P; Lissowska, J; Fabianova, E; Mates, D; Bencko, V; Foretova, L; Janout, V; Brennan, P; Boffetta, P. (2010): Occupational exposure to polycyclic aromatic hydrocarbons and lung cancer risk: a multicenter study in Europe. Occup. Environ. Med., 67: 98-103

Sojinu, OSS; Wang, J.Z; Sonibare, O.O; Zeng, EY. (2010). Polycyclic aromatic hydrocarbons in sediments and soils from oil exploration areas of the Niger Delta, Nigeria," Journal of Hazardous Materials, 174 (1-3): 641-647

Srogi, K. (2007). Monitoring of environmental exposure to polycyclic aromatic hydrocarbons: a review. Environ. Chem. Lett., 5:169-195

Wang, XT; Chen, L; Wang, XK; Lei, BL; Sun, YF; Zhou, J; Wu, MH. (2015). Occurrence, sources and health risk assessment of polycyclic aromatic hydrocarbons in urban (Pudong) and suburban soils from Shanghai in China. Chemosphere, 119: 1224-1232

WHO (2000). Chapter 5.9 PAHs Air Quality Guidelines - Second Edition WHO Regional Office for Europe, Copenhagen, Denmark, 2000 Pp 1 - 24

Zheng, B; Wang, L; Lei, K; Nan, B. (2012). Distribution and ecological risk assessment of polycyclic aromatic hydrocarbons in water, suspended particulate matter and sediment from Daliao River estuary and the adjacent area, China. Chemosphere, 149: 91-100. 